\title{
Preface Issue 3-2012
}

\author{
Hans-Christoph Grunau
}

Published online: 24 July 2012

(C) Deutsche Mathematiker-Vereinigung and Springer-Verlag 2012

Although the problem of finding periodic solutions of Hamiltonian systems is easily explained, it is in many cases quite difficult to solve and in most cases still open. This question-among others - gave rise to the development of symplectic geometry. In this context, Alan Weinstein conjectured in 1978 that every hypersurface of the standard symplectic $\mathbb{R}^{2 n}$ of so called contact type admits a closed orbit. Being of contact type generalises the notion of an object being star-shaped. The survey article of Frederica Pasquotto provides "A short history of the Weinstein conjecture". Some cases of this conjecture have been solved while in its full generality, it is still open.

Herbert von Kaven from Detmold in North Rhine-Westphalia established the eponymous foundation which awards usually every year the von Kaven prize to a young and promising mathematician. The decision is made by the German Research Foundation's (DFG) mathematics review board. During the 2011 annual meeting of "Deutsche Mathematiker-Vereinigung", that year's von Kaven prize was awarded to the DFG-Heisenberg-fellow Christian Sevenheck. His field of research is complex algebraic geometry and in particular mirror symmetry. Sometimes it is possible to find correspondences between two seemingly completely different situations, the so called A-model and the B-model, which may arise e.g. in algebraic geometry and string theory respectively. It may be possible to use results from the B-model to obtain results in the A-model, once these connections have been better understood. Details can be found in Christian Sevenheck's survey article "Mirror symmetry, singularity theory and non-commutative Hodge structures".

H.-Ch. Grunau (凶)

Institut für Analysis und Numerik, Fakultät für Mathematik, Otto-von-Guericke-Universität, Postfach 4120, 39016 Magdeburg, Germany

e-mail: hans-christoph.grunau@ovgu.de 
One year ago, in Issue 3-2011, one could find the survey article by Simon Brendle on "Sphere Theorems in Riemannian Geometry", which was dedicated to Wilhelm Klingenberg who had died on the 14th October 2010. One of the main achievements of Wilhelm Klingenberg was his contribution to proving the topological sphere theorem: If the sectional curvatures of a compact simply connected $n$-dimensional Riemannian manifold are contained in the interval $\left(\frac{1}{4}, 1\right]$, then the manifold is homeomorphic to a sphere. In the current issue, Jost-Hinrich Eschenburg gives a personal view and a number of remarks on further fundamental scientific achievements of Wilhelm Klingenberg, his Ph.D. advisor.

The book reviews section is concerned with new publications on toric varieties, nonabelian algebraic topology and the nonlinear theory of water waves. 